
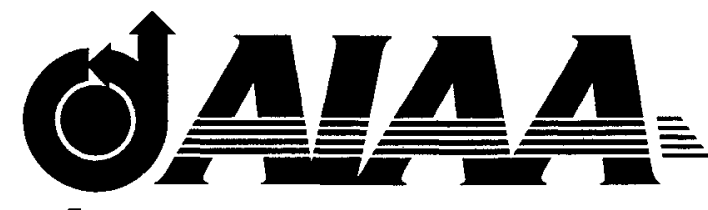

A01-34520

\title{
AIAA 2001-3890 \\ EXPERIMENTAL INVESTIGATION OF HALL THRUSTER INTERAL MAGNETIC FIELD TOPOGRAPHY
}

Peter Y. Peterson, Alec D. Gallimore, and James M. Haas Plasmadynamics and Electric Propulsion Laboratory

University of Michigan

Ann Arbor, MI

\section{$37^{\text {th }}$ AIAA/ASME/SAE/ASEE Joint Propulsion Conference And Exhibit$$
\text { 8-11 July } 2001
$$$$
\text { Salt Lake City, Utah }
$$ 


\title{
EXPERIMENTAL INVESTIGATION OF HALL THRUSTER INTERAL MAGNETIC FIELD TOPOGRAPHY
}

\author{
Peter Y. Peterson*, Alec D. Gallimore ${ }^{\S}$, and James M. Haas ${ }^{* *}$ \\ Plasmadynamics and Electric Propulsion Laboratory \\ Department of Aerospace Engineering \\ The University of Michigan \\ College of Engineering \\ Ann Arbor, MI 48109 USA \\ Phone: 734-764-4199 \\ Fax: $734-763-7158$
}

\begin{abstract}
$\underline{\text { Abstract }}$
Magnetic field measurements were made in the discharge channel of the 5-kW-class P5 laboratory-model Hall thruster to investigate what effect the Hall current has on the static, applied magnetic field topology. The P5 was operated at $1.6 \mathrm{~kW}$ and $3.0 \mathrm{~kW}$ with a discharge voltage of $300 \mathrm{~V}$. The magnetic coils were operated at currents that minimized the discharge current for each power level investigated. A miniature inductive loop probe (B-Dot probe) was employed to measure the radial magnetic field profile inside the discharge channel of the P5 with and without the plasma discharge. These measurements are accomplished with minimum disturbance to thruster operation with the High-speed Axial Reciprocating Probe (HARP) system. Data are presented at axial locations ranging from $1 \mathrm{~cm}$ downstream of the anode to $6 \mathrm{~cm}$ downstream of the thruster exit plane, and at several radial positions covering the width of the discharge chamber. Measured magnetic field profiles are compared to calculated self-field profiles from previously measured Hall current profiles. The results of the B-Dot probe's measurements indicate a change in the magnetic field topography, from that of the vacuum field measurements, in the discharge channel of the P5 Hall thruster at the two thruster power levels investigated.
\end{abstract}

\section{$\underline{\text { Introduction }}$}

As new space missions are planned, the requirements of a spacecraft propulsion system increase beyond the current level of technology. One recently prescribed requirement is for a propulsion system to operate in "dual-mode". Dual-mode is the ability of a thruster to operate efficiently in both a high-thrust and lowspecific impulse (e.g., for orbit transfer operations) mode and a high-specific impulse and low-thrust mode (e.g., for station-keeping) ${ }^{1}$. Other design drivers for advanced space propulsion systems revolve around human space exploration. A piloted mission to Mars for example would require a reliable propulsion system with high power, high performance, and long life.

A favorable candidate for these types of missions is the closed-drift Hall thruster. A Hall thruster is a coaxial plasma device in which an applied magnetic field effectively traps electrons in the discharge channel of the thruster. The electrons are usually emitted from an external cathode while the applied magnetic field is typically created by a magnetic circuit composed of electromagnetic solenoids and pole pieces. The magnetic circuit of a typical Hall thruster produces a radial magnetic field topology at the exit of the discharge channel with peak fields on the order of a few hundred-gauss. If the magnetic field topology in the discharge channel is designed properly, the accelerating ions will experience a focusing effect through what is referred to as a magnetic lens ${ }^{2}$. One of the main characteristics of a Hall thruster is the azimuthally drifting electrons. These electrons form a region of azimuthal current in the discharge channel, which is typically on the order of 5-10 times the discharge current. This estimate is

\footnotetext{
${ }^{*}$ Graduate Student, Aerospace Engineering, Student Member AIAA

$\S$ Associate Professor, Aerospace Engineering and Applied Physics, Associate Fellow AIAA.

${ }^{* *}$ Former Graduate Student, Aerospace Engineering, Currently at Edwards Air Force Base
} 
based on Hall parameters expected in Hall thruster discharge chambers; e.g., 200-300. This estimate can lead to Hall currents on the order of $150 \mathrm{~A}$ for a $5 \mathrm{~kW}$ Hall thruster.

Recent plasma measurements inside the discharge channel of the P5 indicate that the true, effective Hall parameter ranges from 10-20 near the anode to approximately 1000 in the acceleration region ${ }^{4}$. These results suggest that the Hall current may be larger than previously thought, and that its induced magnetic field could impact the applied magnetic field topology significantly. As the magnitude of the Hall current increases at higher thruster power levels, the effects of the self-magnetic field induced by the Hall current may become more important. As the self-magnetic field magnitude increases, its effect on the magnetic circuit applied field topology is greater, thus decreasing the chances of maintaining the desired magnetic lens profile in the discharge channel. In designing next-generation Hall thrusters, the magnetic field topology for a given magnetic circuit design can be predicted with great precision by using 3-D magnetostatic computer codes. However, to accurately predict the magnetic field topology during thruster operation, a better understanding of the effects of the magnetic field induced by the Hall current is needed.

The University of Michigan Plasmadynamics and Electric Propulsion Laboratory (PEPL) has endeavored in the past to develop and improve plasma diagnostic techniques to be used on plasma propulsion systems $^{5-12}$. An attempt to map the magnetic field topology near the exit of a D-55 Anode Layer Thruster was performed at PEPL ${ }^{12}$. However, these measurements were limited to $15 \mathrm{~mm}$ downstream of the exit plane due to the large perturbations in thruster discharge current that ensued when the Hall probe was placed closer to the engine. It was concluded that the Hall probe was entering the Hall current region and thus disrupting the stable operation of the thruster. To continue the research effort initiated by the D-55 verynear-field investigation, and to gather knowledge on the effects of the self-induced magnetic field at high power operation of a stationary plasma thruster, a miniature inductive loop probe (B-Dot probe) system was developed and used in conjunction with the PEPL High-speed Axial Reciprocating Probe (HARP) system to map the radial magnetic field of the P5 Hall thruster.

The University of Michigan's HARP system was designed to address concerns associated with placing probes within an operating Hall thruster. These concerns include probe life and thruster perturbation. These concerns are addressed by the high sweeping speed of the HARP system (described below). The HARP system also provides a unique opportunity to incorporate a time response B-Dot probe by virtue of its motion. B-Dot probes are typically used for pulsed and inductive plasma discharges with time-varying magnetic fields $13,14,15$. However, by combining the high speed of the HARP table $(\mathrm{dx} / \mathrm{dt})$ with the B-Dot probe response to a time-varying magnetic field $(\mathrm{dB} / \mathrm{dt})$, one can measure a change in a magnetic field magnitude as the probe is swept $(\mathrm{dB} / \mathrm{dx})$ into the discharge channel of the thruster.

This work presented here does not represent the first time an inductive loop probe has been used on a Hall thruster. Barkalov incorporated two inductive loops into a pulsed Hall thruster to measure the location and magnitude of the Hall current ${ }^{16}$. The two coils were located along the inner and outer walls of the ceramic discharge channel. The Hall current was determined to be a maximum of approximately $30 \mathrm{~A}$, for a $300 \mathrm{~V}$ 15 A plasma discharge, at a axial position of $45 \mathrm{~mm}$ downstream of the anode and $10 \mathrm{~mm}$ from the inner wall of the investigated pulsed Hall thruster. The Hall thruster investigated in this reference had an outer diameter of $160 \mathrm{~mm}$, a width of $30 \mathrm{~mm}$, and a depth of $60 \mathrm{~mm}$.

This paper will briefly describe the experimental facilities, the Hall thruster, and the HARP system. The theory of inductive loop operation is reviewed as are the construction, set-up, and calibration of the BDot system. Finally, thruster magnetic field profiles both in vacuum and in the presence of the plasma discharge are presented and discussed.

\section{Experimental Apparatus}

\section{Vacuum Facilities}

All the experiments were conducted in the University of Michigan's Large Vacuum Test Facility (LVTF) that has a diameter of 6 meters and a length of 9 meters. The LVTF chamber is evacuated by two 2,000 CFM blowers and four $400 \mathrm{CFM}$ mechanical pumps to moderate vacuum ( 30 - $100 \mathrm{mTorr})$. To reach high vacuum the LVTF is equipped with seven CVI TM-1200 cryopumps, with a combined pumping speed of $\sim 500,000 \mathrm{l} / \mathrm{s}$ on air, and $\sim 240,000 \mathrm{l} / \mathrm{s}$ on xenon and a base pressure in the high $10^{-8}$ Torr range. The cryopump system can be operated with any number of pumps in use. For the experiments reported here the LVTF was operated with only four 
cryopumps to match operating conditions of prior experiments ${ }^{4,17}$. At the four-cryopump configuration, the combined pumping speed of $140,000 \mathrm{l} / \mathrm{s}$ on xenon with a base pressure $1.6 \times 10^{-7}$ Torr was achieved. At a $10.2 \mathrm{mg} / \mathrm{s}$ anode mass flow and a $0.6 \mathrm{mg} / \mathrm{s}$ cathode mass flow the operating pressure of the LVTF was $8.5 \times 10^{-6}$ Torr (xenon).

The schematic of the LVTF is shown in Figure 1. The Hall thruster was mounted in the center of the vacuum chamber on a X-Y computer-controlled linear positioning system.

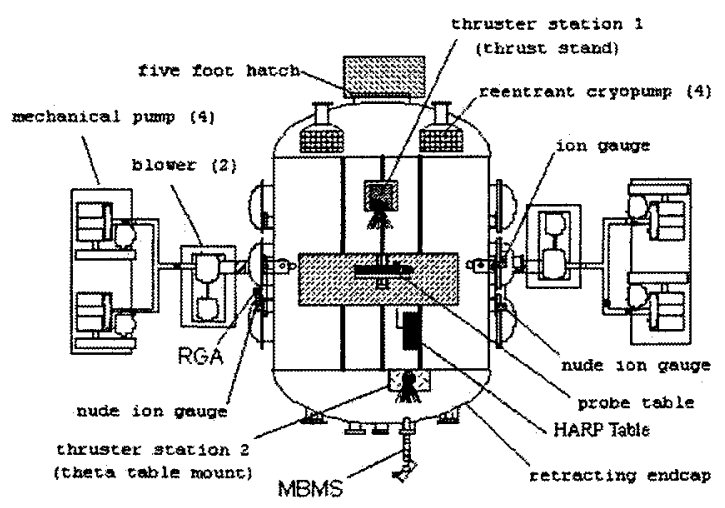

Fig. 1 Schematic of the LVTF.

Hall Thruster

The experimental results presented in this paper were conducted on the 5-kW-class laboratory model P5 Hall thruster. The P5 Hall thruster was developed by the University of Michigan and the Air Force Research Laboratory to serve as a test-bed for new diagnostics and for investigating Hall thruster processes. Depicted in Fig. 2, the P5 has an outer diameter of $170 \mathrm{~mm}$, a channel width of $25 \mathrm{~mm}$, and a channel depth of $38 \mathrm{~mm}$.

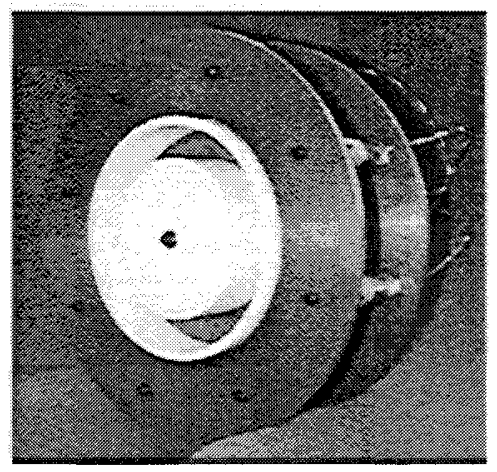

Fig. 2 P5, $5 \mathrm{~kW}$ class laboratory model Hall thruster.
The P5 performance characteristics, presented in a previous work $^{18}$, are comparable to commerciallyavailable $5 \mathrm{~kW}$ thrusters. Table 1 shows the measured performance characteristics of the P5 for the two operating conditions used in this experiment. The hollow cathode used for these test was provided by Moscow Aviation Institute (MAI). The cathode provides thermally emitted electrons to the discharge by a small disk of lanthanum hexaboride $\left(\mathrm{LaB}_{6}\right)$.

\begin{tabular}{|l|c|c|l|l|l|}
\hline Case & $\begin{array}{c}\text { Vd } \\
{[\mathrm{V}]}\end{array}$ & $\begin{array}{c}\text { Id } \\
{[\mathrm{A}]}\end{array}$ & $\begin{array}{c}\text { Power } \\
{[\mathrm{kW}]}\end{array}$ & $\begin{array}{c}\text { Total } \\
\text { Specific } \\
\text { Impulse } \\
{[\mathrm{sec}]}\end{array}$ & $\begin{array}{c}\text { Total } \\
\text { Efficiency }\end{array}$ \\
\hline$(1)$ & 300 & 5.4 & 1.6 & 1550 & $48 \%$ \\
\hline$(2)$ & 300 & 10.1 & 3 & 1650 & $51 \%$ \\
\hline
\end{tabular}

Table 1 P5 thruster performance range for the experiments discussed in this work ${ }^{18}$

High-speed Axial Reciprocating Probe

The HARP system consists of a LM1210 high-speed linear motor and encoder manufactured by Trilogy. The linear encoder provided a linear resolution of 5 microns to a Pacific Scientific SC950 digital brushless servo drive controller. A computer controls the position, speed, acceleration, deceleration, and sweep configuration for the HARP system. The linear table was placed within a stainless steel shroud encased with graphite plates (Figs. 3 and 4).

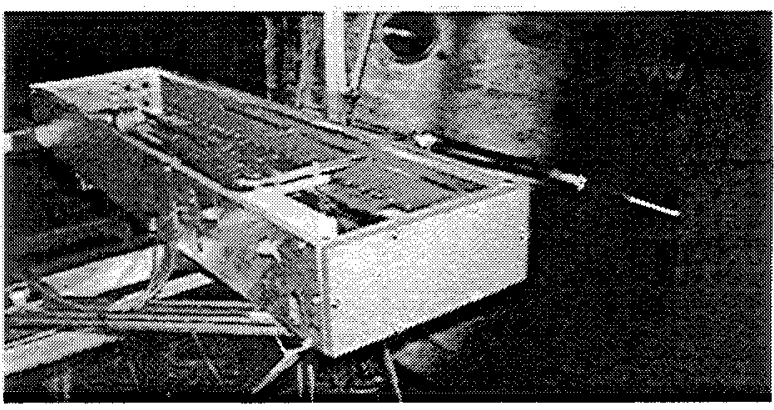

Fig. 3 PEPL's High-speed Axial Reciprocating Probe (HARP) system. The top cover of the shroud has been removed to show the linear motor and B-Dot integrator. 


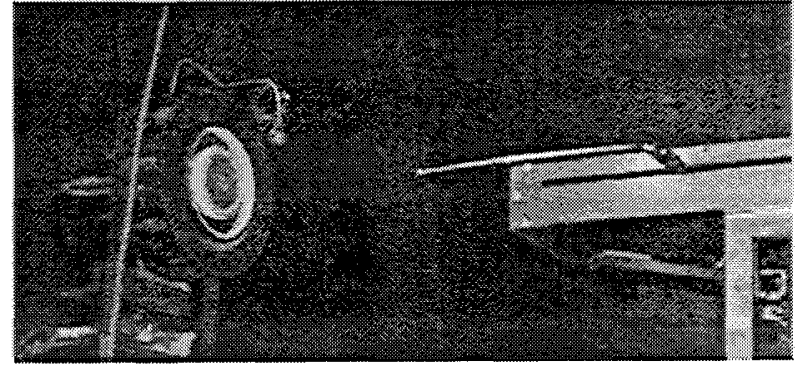

Fig. 4 Typical configuration of the HARP table and the $P 5$ thruster. The picture shows the B-Dot probe in its zero position, which is $150 \mathrm{~mm}$ downstream of the thruster exit plane. A sweep of the probe occurs over $180 \mathrm{~mm}$.

The primary issue involving internal probe measurements of a Hall thruster is the ability of the probe to survive. Ablation of probe material also affects thruster operation. Therefore, the main driving factor in determining the maximum resonance time that a probe can remain in the discharge channel of a thruster is the characteristic time for probe material ablation. The ablation time for $99 \%$-pure alumina has been determined to be approximately $150 \mathrm{~ms}$ for a 5 $\mathrm{kW}$ plasma discharge $\mathrm{e}^{4}$.

The time that the B-Dot probe spent in the discharge channel for these experiments was approximately 80 $\mathrm{ms} \pm 10 \mathrm{~ms}$. Position versus time of the B-Dot probe is presented in Fig. 5. The peak velocity and acceleration of the HARP for these experiments was approximately $\pm 5.5 \mathrm{~m} / \mathrm{s}$ and 130 to $-100 \mathrm{~m} / \mathrm{s}^{2}$, respectively.

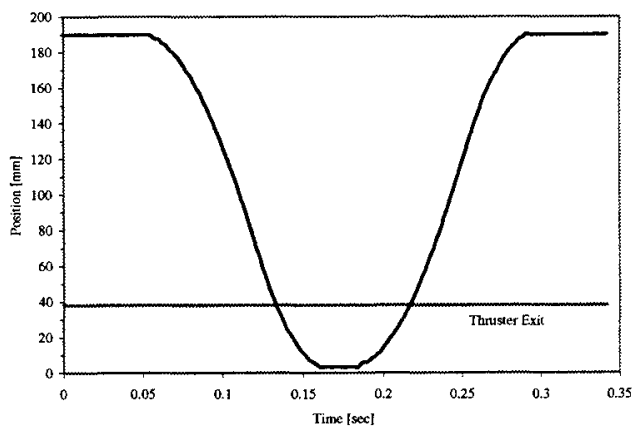

Fig. 5 Position of the B-Dot probe as a function of time.

Inductive Loop Probe

To measure the internal magnetic field topography of an operating Hall thruster, there were two methods that were considered. The first method was a Hall probe, and the second was an inductive loop probe. Four criteria were established to determining the practicality of both methods. The criteria that were considered were size, thermal response, fabrication issues, and cost. The size of the magnetic field probes that could be constructed from each method was comparable. The thermal response of each of the potential methods should not be a factor due the duration that a probe would spend in the plasma discharge, as discussed in the description of the HARP positioning system. The fabrication of a Hall probe system would have been less complex in assembly than the winding of the inductive coil for the B-Dot system. The cost of the miniature magnetic field probe was the deciding factor in choosing which probe system would be used for this investigation. Due to the nature of the HARP system and the size of the discharge channel of the P5 thruster, there was always the possibility that the probe would come into contact with the thruster's channel walls or the anode itself, due to small misalignments of the thruster. Considering the cost of miniature Hall probes, this avenue of mapping the magnetic field of the discharge channel was turned down in favor of the B-Dot system.

The inductive loop probe is a well-established plasma diagnostic technique for time-varying magnetic fields. Typical plasma discharges that make use of the B-Dot probing technique include pulsed and inductive plasmas $^{13,14,15}$. The basic operating principle of the BDot probe stems from the observation that current is induced in a conducing coil immersed within a timevarying magnetic field. The ensuing output voltage from the coil is proportional to its cross-sectional area (A), the number of turns in the coil $(\mathrm{N})$, and the time characteristic of the magnetic field $(\mathrm{dB} / \mathrm{dt})$. The expression of the voltage output from a B-Dot probe is given as Eq. (1).

$$
V=n A^{d B} / d t
$$

Since Hall thruster typically operates with a steady applied magnetic field, direct application of the B-Dot probe is problematic. To circumvent this issue, the HARP position system was used to provide a timevarying magnetic field signal by virtue of moving the probe into (and out of) the applied, steady magnetic field. Therefore, by combining the motion of a highspeed table $(\mathrm{dx} / \mathrm{dt})$ with the integrated signal of a $B$ Dot probe in a time-varying magnetic field $(\mathrm{dB} / \mathrm{dt})$, one is able to measure the DC applied magnetic field as the probe is swept into and out of the Hall thruster discharge channel. 
A dual-supply integrator that incorporates an Analog Devices AD549 ultra-low input bias current operational amplifier and a AD624 precision instrumentation amplifier, was chosen to integrate the $\mathrm{B}$-Dot probe raw signal. The expression of the integrated output voltage from a B-Dot probe is shown in Eq. (2)

$$
V=\frac{n A}{R C G} B
$$

Where the $G$ is the amplifier gain, $R C$ is the integrators resistor and capacitor, respectively, and $\mathrm{B}$ is the measured magnetic field. The amplified voltage output from the B-Dot probe integrator circuit was recorded with a Tektronix TDS 540 digital oscilloscope in high-resolution acquisition mode. The data was then downloaded to a computer for processing. A diagram of the integrator circuit used in the experiments described in this paper is shown in Fig. 6.

The B-Dot probe support structures used for this investigation were constructed of $99 \%$ alumina ceramic tubes and ceramic paste. The inductive loops were wound with 38 and 40 gauge magnetic wire with enamel nonconductor coatings. The coils used in this investigation were wound around 1.6 to $2.5 \mathrm{~mm}$ diameter alumina ceramic tubes 2.2 to $2.5 \mathrm{~mm}$ in length. The number of turns in the probe coils ranged from 89 to 136 . The final dimensions of the B-Dot probes used in this investigation ranged from 4 to 4.3 $\mathrm{mm}$ in diameter and 4 to $4.2 \mathrm{~mm}$ in length

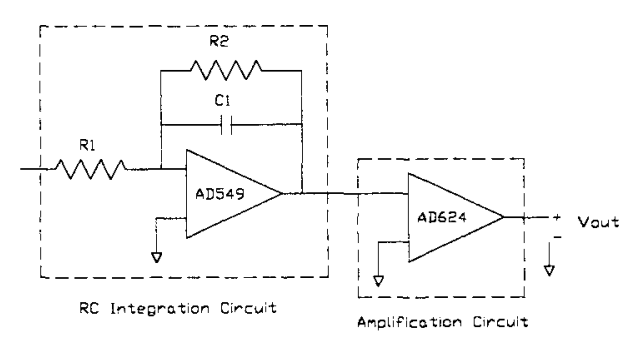

Fig. 6 B-Dot Integrator and Amplifier circuit. Where $\mathrm{R} 1$ and $\mathrm{C} 1$ are the primary components integration.

Figure 7 shows the mapped area inside the discharge channel of the P5 thruster. The B-Dot probe was axially swept from $150 \mathrm{~mm}$ downstream of the exit plane to $10 \mathrm{~mm}$ in downstream of the anode. This axial sweep profile was repeated for three radial positions, $3.81 \mathrm{~mm}$ from the inner wall, the centerline (at $12.7 \mathrm{~mm}$ ), and $3.81 \mathrm{~mm}$ from the outer wall of the discharge channel. In an attempt to minimize the heating of B-Dot probe between sweeps, the probe home position was set to $152 \mathrm{~mm}$ downstream of the thruster exit plane. The cathode plane was located 50 $\mathrm{mm}$ downstream of the thruster exit plane and was oriented $45^{\circ}$ counter-clock wise from the plane of plane mapped The results presented in this paper will cover the first $100 \mathrm{~mm}$ downstream of the anode.

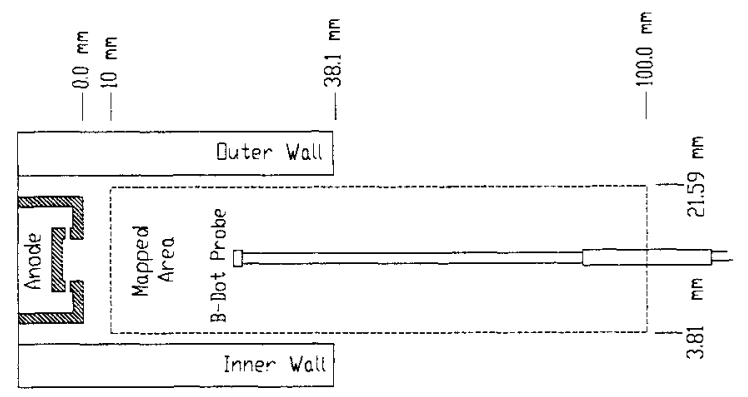

Fig. 7 B-Dot probes mapped area inside and outside of the P5 discharge channel.

\section{Results and Discussion}

Calibration of B-Dot Probe

The initial goal of this experiment was to calibrate the B-Dot probe and integrator circuit with an electric and magnetic field $(\mathrm{ExB})$ source. The idea behind the ExB source was to approximate, under a controlled and understood manner, the fields that the B-Dot probe would experience when the probe is traveling through the discharge channel of an operating thruster. The ExB source was comprised of two electromagnetic coils, to provide the magnetic field, and two parallel plates for the electric field. The configuration of the ExB source can be seen in Fig. 8.

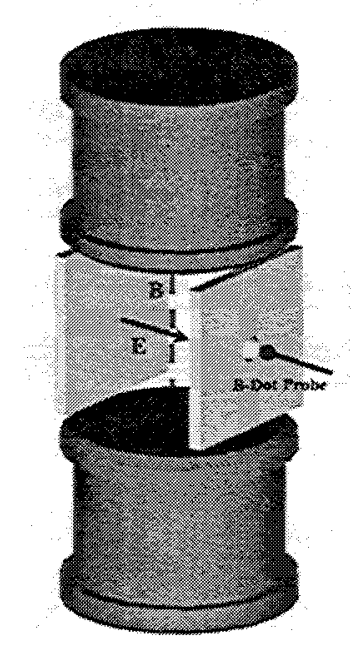

Fig. 8 The ExB source configuration. 
The magnetic field of the ExB source was first mapped using a NIST-traceable Walker Scientific MG-5DAR Hall probe. Then the B-Dot probe and HARP systems were configured to measure the ExB source with either the applied magnetic and/or electric fields. All parameters of the B-Dot probe and HARP, such as speed, acceleration, deceleration, probe sweep length, and transmission line length that would be used during experiments with the P5 were used in the calibration runs.

A Kikusui power supply provided a constant current of 9 A to the ExB source to drive a 167 Gauss traverse magnetic field in the center of the ExB source. A Kepco Bipolar power supply was used to apply a 15 $\mathrm{kV} / \mathrm{m}$ electric field across the plates that were used to drive the electric field. This electric field magnitude was chosen to represent the typical value expected in $300 \mathrm{~V}$ Hall thruster discharge chambers. However, the peak electric field, which resides in a small region of the discharge chamber at the onset of the acceleration zone, can reach $25 \mathrm{kV} / \mathrm{m}^{4,17}$.

The choice in the ExB source's electric field magnitude for the calibration of the B-Dot probe was believed to be of sufficient magnitude to represent the electric field of an operating 300 volt Hall thruster. However, after the experimental phase of this investigation was completed the initial calculation of the possible electric field that the B-Dot probe could experience was determined to be greater than was previously calculated ${ }^{4,17}$. The P5 Hall thruster operating at 300 volts and 5.4 A plasma discharge has an electric field, that was measured with a emissive probe, of a maximum of $24 \mathrm{kV} / \mathrm{m}$. The P5 operating at 300 volts and $10 \mathrm{~A}$ discharge had a maximum electric field of $20 \mathrm{kV} / \mathrm{m}$. The $15 \mathrm{kV} / \mathrm{m}$ that was used during the calibration of the B-Dot probes should still indicate if the B-Dot probes are influenced by the presence of a steep electric field.

Figure 9 shows the measured magnetic field profile of the ExB source as measured by the B-Dot probe in the absence of an applied electric field while Figure 10 presents the same measurements in the presence of an applied electric field of $15 \mathrm{kV} / \mathrm{m}$. The measured data presented in Figure $9 \& 10$ are actual five sets of BDot traces averaged together at the same ExB source input settings. Figures $9 \& 10$ also contain the Hall probe traces of the ExB source at the two investigated conditions.

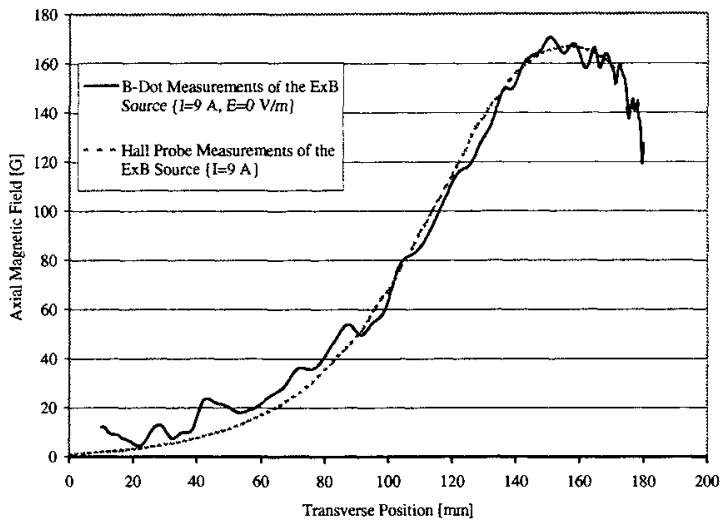

Fig. 9 B-Dot and Hall probe measurements of the ExB source with no applied electric field $\left\{I \_c o i l=9\right.$ A\}.

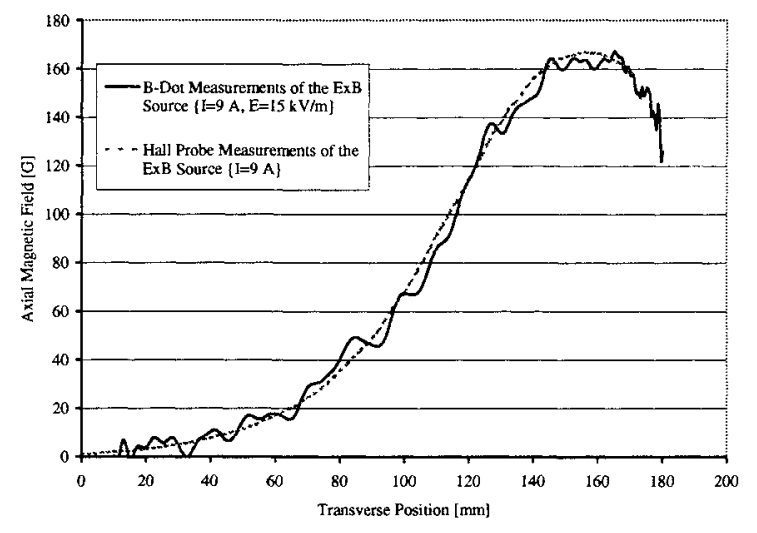

Fig. 10 B-Dot and Hall probe measurements of the ExB source with an applied electric field of 15 kV/m \{I_coil = 9 A, $\}$.

As can be seen in Figs. 9 and 10, the B-Dot probe captured the profile and magnitude of the ExB source. The presence of the $15 \mathrm{kV} / \mathrm{m}$ electric field had no noticeable effect on the measured magnetic field of the B-Dot probe.

Vacuum Field Measurements with B-Dot Probe The vacuum magnetic field profiles of the P5 thruster were mapped using the B-Dot and HARP systems along the radial and axial positions described earlier. Two thruster power levels were investigated; $1.6 \mathrm{~kW}$ and $3.0 \mathrm{~kW}$, both at a discharge voltage of $300 \mathrm{~V}$. The inner and outer electromagnet coils were operated in a manner that minimized the discharge current of the thruster. Once these coil currents were determined for both power levels, the P5 discharge was extinguished while the magnets were left on for vacuum field measurements.

The B-Dot and Hall probe vacuum field measurements of the P5 at coil current settings for 1.6 
$\mathrm{kW}$ and $3.0 \mathrm{~kW}$ are shown in Figs 11 and 12 , respectively. The thruster exit plane and the midplane of the magnetic circuit outer and inner front poles are labeled in these figures. Once again, each BDot profile presented represents the average of 5 or more data sweeps. All trends observed in individual B-Dot sweeps are captured in the average profile.
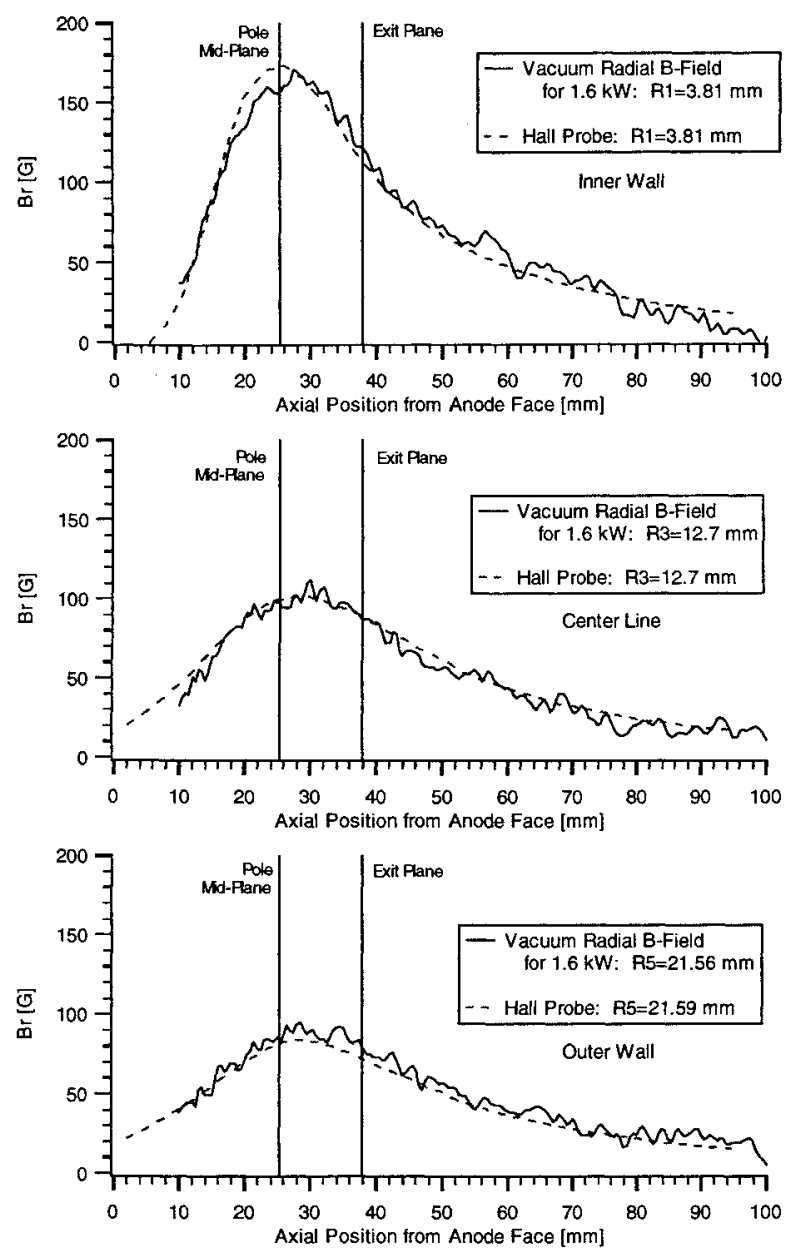

Fig. 11 Vacuum magnetic field profiles of the P5 at $1.6 \mathrm{~kW}\left\{\mathrm{I} \_\right.$in $=2 \mathrm{~A}, \mathrm{I} \_$out $\left.=1 \mathrm{~A}\right\}$
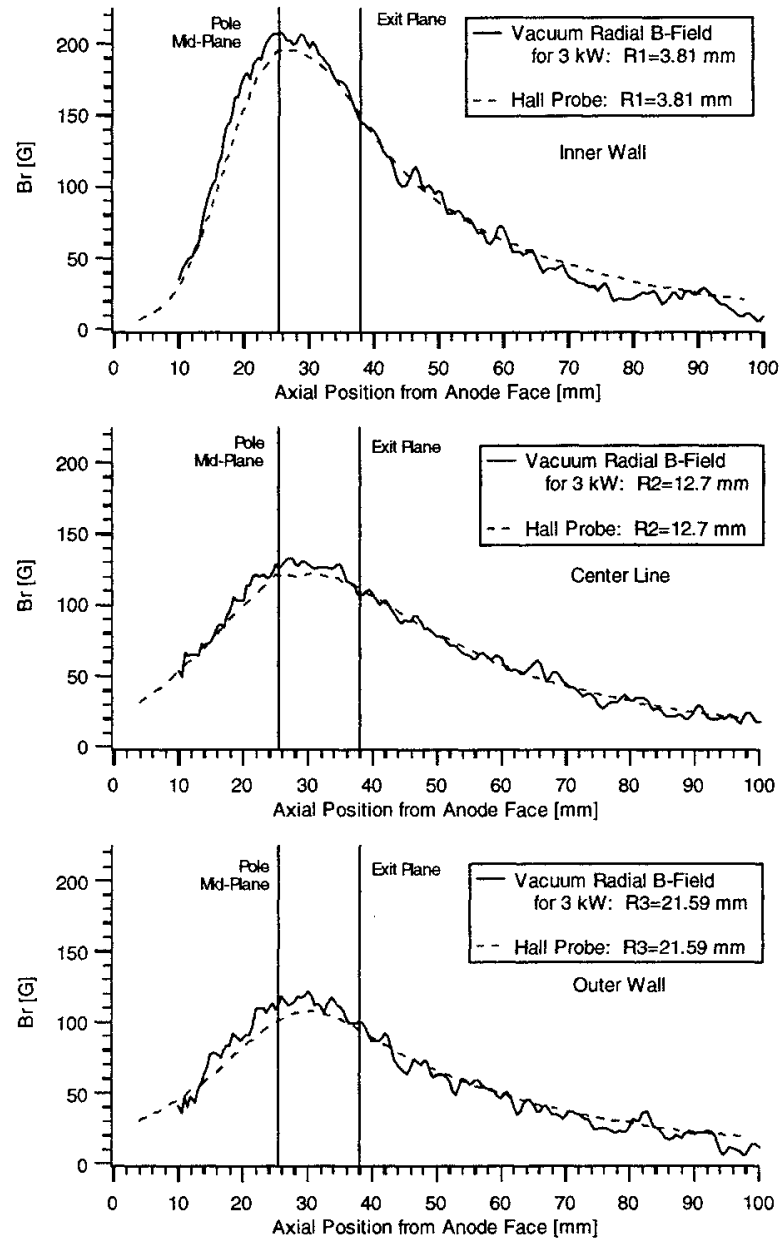

Fig. 12 Vacuum magnetic field profile of the P5 at $3.0 \mathrm{~kW}\left\{\mathrm{I} \_\right.$in $=3 \mathrm{~A}, \mathrm{I} \_$out $\left.=2 \mathrm{~A}\right\}$

The figures shows that magnetic field profiles measured with the B-Dot probe match those of the NIST-traceable Hall probe for both coil settings.

Magnetic Field Measurements with Discharge

The discharge channel radial magnetic field with the P5 thruster operating at $1.6 \mathrm{~kW}$ and $3.0 \mathrm{~kW}$ was mapped at three radial positions. The P5 was operated for a minimum of forty minutes before B-Dot sweeps were made to allow the engine to reach thermal equilibrium. This step was conducted to ensure that any anomalies measured with the B-Dot probe were not due to thermal expansion of the magnetic circuit components throughout the duration of the experiment. The results of the B-Dot probe sweeps for the $1.6 \mathrm{~kW}$ thruster power level (300 volt, $5.4 \mathrm{~A}$ ) are shown in Fig. 13. Figure 13 also contains a plot of the thruster discharge current as a function of probe 
position and the corresponding vacuum Hall probe magnetic field measurements.
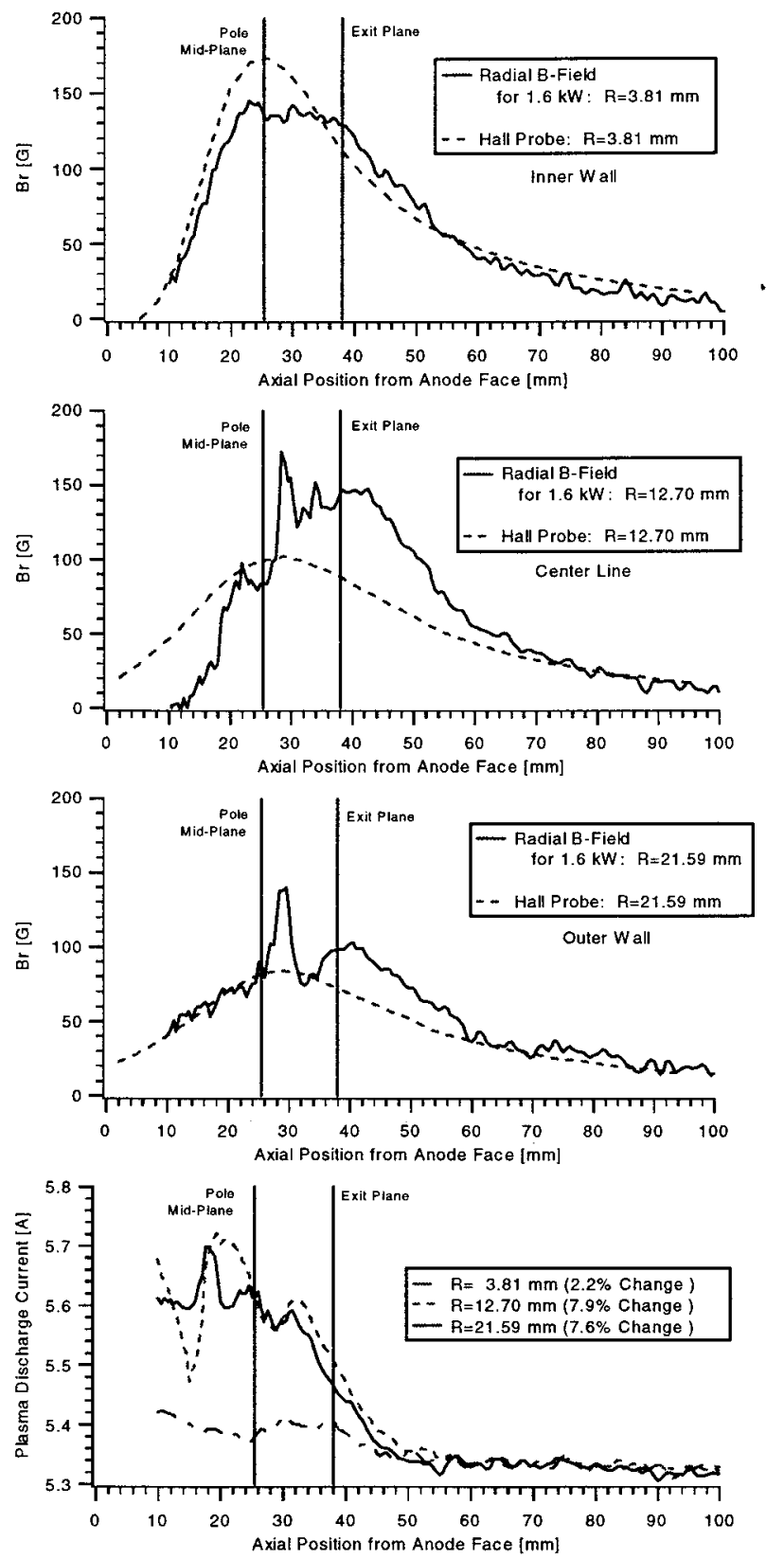

Fig. 13 B-Dot probe magnetic field data at $1.6 \mathrm{~kW}$ and thruster discharge current as a function of probe position.

As can be seen in Fig. 13, the magnitude and profile of the magnetic field in the discharge channel of the P5 Hall thruster is affected by the presence of the plasma discharge.
B-Dot probe, vacuum Hall probe, and thruster discharge current data for the $3.0 \mathrm{~kW}$ power level are shown in Fig. 14.
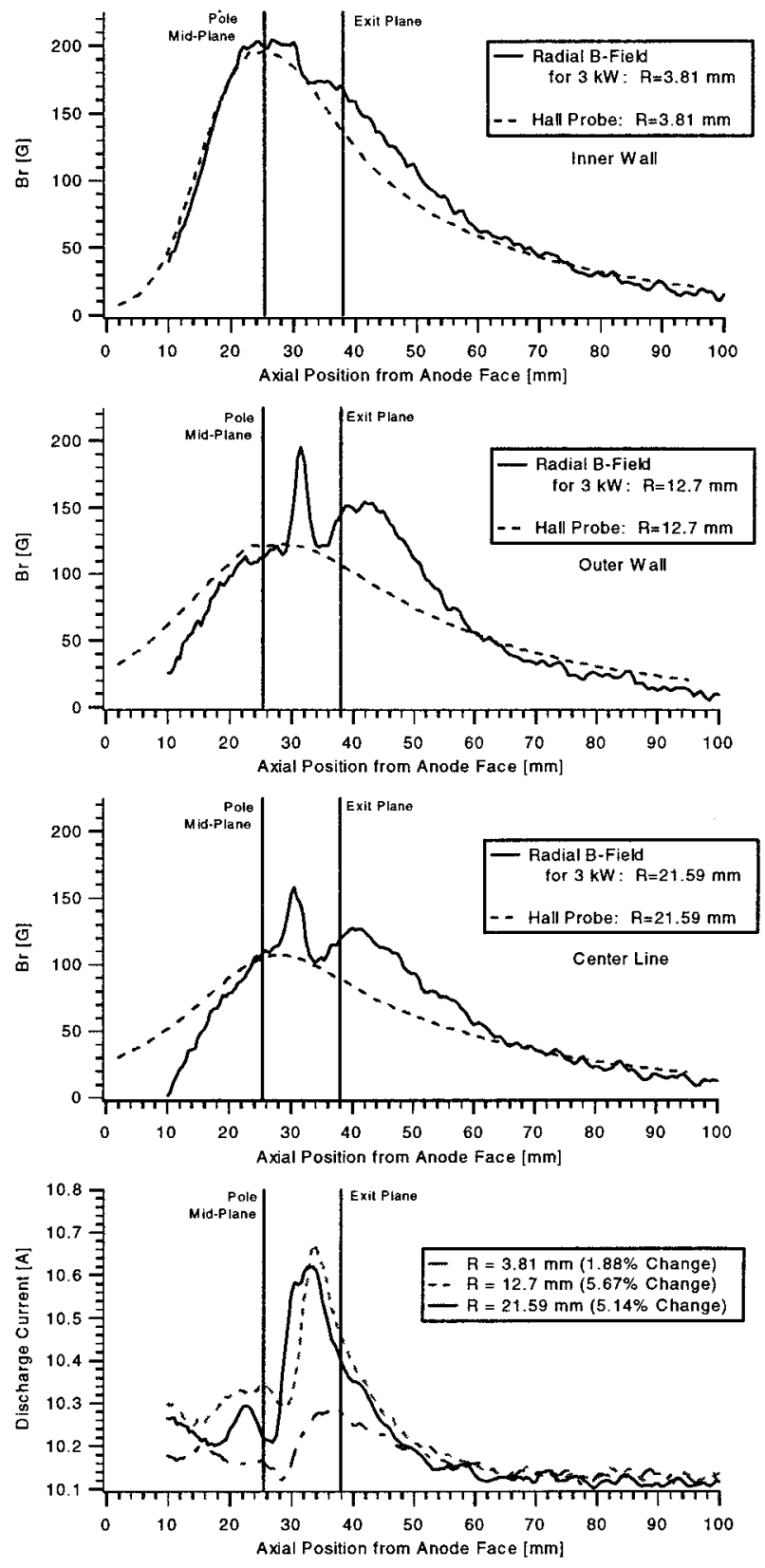

Fig. 14 B-Dot probe magnetic field data at $3.0 \mathrm{~kW}$ and thruster discharge current as a function of probe position.

Once again the B-Dot profiles with the thruster operating at $3.0 \mathrm{~kW}$ differ from those in vacuum at the identical magnet settings. There are several possible explanations to account for this measured difference 
in the magnetic field profiles. The first and foremost explanation, assuming that the B-Dot probe is functioning properly, is the effect of the azimuthal electron drift (Hall current) self magnetic field. As discussed earlier, the Hall current of a closed-drift thruster can be 5 to 10 times the discharge current ${ }^{3}$ or in light of recent internal plasma parameter measurements ${ }^{4}$, possibly much higher.

In both the $1.6 \mathrm{~kW}$ and $3.0 \mathrm{~kW}$ case we see that the BDot probe profiles with the discharge on closely match the vacuum field profiles along the inner wall of the discharge channel. However, the profiles are significantly different near the outer wall of the discharge channel and particularly along the center of the channel.

The largest differences in profiles occur slightly downstream of the thruster exit plane. In the $1.6 \mathrm{~kW}$ results, there is a spike in the measured magnetic field $10 \mathrm{~mm}$ inside of the discharge channel. A characteristic spike exists in the $3.0 \mathrm{~kW}$ data as well however in this case the spike is $7 \mathrm{~mm}$ inside the thruster channel. Neither of these spikes nor the large increases in the B-Dot magnetic field profiles corresponds to locations of peak electric fields in the thruster ${ }^{4}$. The peak electric fields of the P5 are located approximately $5 \mathrm{~mm}$ inside the discharge channel for the $1.6 \mathrm{~kW}$ thruster power level and approximately 2 $\mathrm{mm}$ inside the channel for the $3.0 \mathrm{~kW}$ power level ${ }^{17}$.

Thruster discharge current recorded as the probe entered the discharge channel (Figs. 13 and 14). The maximum disturbance to the thruster discharge current was $8 \%$; during the centerline sweep at the $1.6 \mathrm{~kW}$ power level. The axial region where the greatest perturbation in the thruster discharge current was recorded corresponds with where drops in the magnetic field profiles for the three radial sweeps at $3.0 \mathrm{~kW}$ occur. This can also be seen in the outer wall sweep of the $1.6 \mathrm{~kW}$ case. A possible explanation for these features is that the probe is blocking the natural path of the Hall current in the thruster channel. This explanation can also be argued by the greater perturbation to the thruster discharge current for the centerline and outer wall sweeps. This area of the discharge channel is believed to be the location of the majority of the Hall current for the P5 thruster ${ }^{4}$.

Another possible explanation that is less likely is thermal expansion of the magnetic circuit. However, this prospect was investigated by measuring the vacuum field profiles of the thruster before and immediately after the thruster was operated for an extended amount of time. No discernable changes in the vacuum field profiles were observed during the analysis of the data (not shown).

One final possibility for the measured difference in the results from the vacuum and plasma discharge profiles is that the B-Dot probe output voltage is perturbed by the high electric field in the discharge channel of the thruster. Several precautions were addressed during the fabrication and calibration of the B-dot probe system. The first was to place the integrator circuit as near as possible to the probe, which meant that the integrator was located in the shroud that covers the HARP table (Fig. 3). There was no indication from the baseline sweeps of the HARP table and the B-Dot system of electrostatic interference in the probe or the circuit from the operation of the HARP table alone. Another precaution addressed was to add a low resistance to the output of the B-Dot probe through the selection of the transmission lines and circuit design, thus effectively providing the B-Dot probe with a voltage divider for any unavoidable electrostatic pickup ${ }^{13}$. The final precaution was to calibrate the BDot system with an ExB source as described earlier. It was shown in Fig. 10 that as the B-Dot probe swept the ExB source with a known applied magnetic and electric fields there was no indication of the probe being affected by the presence of a large electric field.

\section{Conclusions}

The magnetic field topography of the P5 Hall thruster was successfully mapped during operation of the thruster with a plasma discharge. The experimentally mapped magnetic field profiles along the width of the discharge channel of a Hall thruster deviated from the vacuum field profile, as measured with a Hall probe. The largest increase observed was approximately $70 \mathrm{G}$ on the centerline sweep of the $1.6 \mathrm{~kW}$ operation condition. These results were accomplished by the development of a new PEPL HARP system internal plasma diagnostic technique with the incorporation of a B-Dot probe. The influence of a Hall thruster's azimuthal electron drift current had been suspected in the past as having little or no affect on the applied field of a Hall thruster. However, this investigation suggests that further research on the influence of a Hall thruster self-magnetic field needs to be addressed, especially in the development of high power Hall thrusters. 


\section{Acknowledgements}

The authors would like to acknowledge the support of this research by NASA through contract NGT3-52348 (Robert Jankovsky, technical monitor). The authors would also like to thank the Air Force Research Laboratory for their assistance in the design and fabrication of the P5 Hall thruster, Dr. Sergi Khartov at $\mathrm{MAI}$ for the use of the $\mathrm{LaB}_{6}$ cathode, and the graduate students at PEPL for their assistance in setting up and running the experiments for this investigation. The authors would also like to thank the technical support staff of the Aerospace Engineering Department.

\section{References}

1. Butler, G.W., Yuen, J.L., Tverdokhlebov, S.O., Semenkin, A.V., Kochergin, A.V., Solodulthin, A.E., Jankovsky, R.S., "Multimode, High Specific Impulse Hall Thruster Technology," AIAA-00-3254, 36th Joint Propulsion Conference (JPC) Huntsville, AL, July 16-19, 2000.

2. Gavryshin, et al., "Physical and Technical Bases of the Modern SPT Development," IEPC-95-38

3. Kim, V., "Main Physical Features and Process Determining the Performance of Stationary Plasma Thrusters", Journal of Propulsion and Power, Vol. 14, No. 5, September-October 1998

4. Haas, J.M.,"Low-perturbation Interrogation of the Internal and Near-field Plasma Structure of a Hall Thruster Using a High-Speed Probe Positioning System," Ph.D. Dissertation, University of Michigan, 2001.

5. Haas, J.M., Gallimore, A.D., McFall, K., and Spanjers, G., "Development of a High-Speed, Reciprocating Electrostatic Probe System for Hall Thruster Interrogation," Review of Scientific Instruments, Vol. 71, No. 11, pp. 4131-4138, November 2000.

6. Williams, G., Smith, T., Gallimore, A.D., "FMT2 Discharge Cathode Erosion Rate Measurements via Laser Induced Fluorescence," AIAA-00-3663, 36th Joint Propulsion Conference (JPC) Huntsville, AL, July 16-19, 2000 .
7. Beal, B.E., Haas, J.M., Gallimore, A.D., "Plasma Density Measurements Inside a Laboratory Model Hall Thruster Using a Resonance Probe Diagnostic," ICOPS-00-4A03, 27th IEEE International Conference on Plasma Science (ICOPS) New Orleans, LA June 4-7, 2000.

8. Hofer, R.R., Haas, J.M., and Gallimore, A.D., "Development of a 45-Degree Parallel-Plate Electrostatic Energy Analyzer for Hall Thruster Plume Studies: Preliminary Data," IEPC-99-113, 26th International Electric Propulsion Conference, Kitakyushu, Japan, October 1999.

9. Gulczinksi, F.S., Hofer, R.R., and Gallimore A.D., "Near-field Ion Energy and Species Measurements of a $5 \mathrm{~kW}$ Laboratory Hall Thruster, " AIAA-99-2430, 35th Joint Propulsion Conference, Los Angeles, CA, June 1999.

10. Kim, S., and Gallimore, A.D., "Plume Study of a $1.35 \mathrm{~kW}$ SPT-100 Using an ExB Probe, " AIAA99-2423, 35th Joint Propulsion Conference, Los Angeles, CA, June 1999.

11. King, L. B., and Gallimore, A. D., "A Gridded Retarding Pressure Sensor for Ion and Neutral Particle Analysis in Flowing Plasmas," Review of Scientific Instruments, Vol. 68, No. 2, Feb. 1997, 1183-1188.

12. Domonkos, M. T., Gallimore, A. D., Marrese, C. M., and Haas, J. M., "Very Near-Field Plume Investigation of the Anode Layer Thruster," Journal of Propulsion and Power (AIAA), Vol. 16, No. 1, January-February 2000.

13. Lovberg, R. H., "Plasma Diagnostic Techniques," Chapter 3, Academic Press Inc., 1965

14. Piejak, R., Godyak, V., Alexandrovich, B., "The electric field and current density in a lowpressure inductive discharge measure with BDot probes," Journal of Applied Physics, American Institute of Physics, Vol. 81, No. 8, April 1997

15. Black, D.C., "Direct Magnetic Field Measurements of Flow Dynamics and MicroTurbulence Enhanced Electron Collisionality in Magnetized Coaxial Accelerator Channels," 
Ph.D. Dissertation, North Carolina State University, 1996.

16. Barkalov, E.E., Veselovzorov, A.N, Subbotin, M.L., "Expermential study of the azimuthal electron drift current in Hall accelerators," Sov. Phys. Tech. Phys., Vol. 35, No. 2, February 1990

17. Haas, J.M., and Gallimore, A.D., "Characterization of the Internal Plasma Structure of a $5 \mathrm{~kW}$ Hall Thruster," IEPC-99078, 26th International Electric Propulsion Conference, Kitakyushu, Japan, October 1999.

18. Haas, J. M., Gulczinski, F. S., Gallimore, A. D., Spanjers, G.G., Spores, R.A., "Performance Characteristics of a $5 \mathrm{~kW}$ Laboratory Hall Thruster," AIAA-98-3503, 34th Joint Propulsion Cleveland, OH, July 12-15, 1998. 